\title{
Determination of contrast factors for cubic slip-systems and their application in the microstructural characterization of binary $\mathbf{F m} \overline{3} \mathbf{m}$ materials
}

\author{
Danyel Cavazos-Cavazos ${ }^{1}$ and Flavio F. Contreras-Torres ${ }^{1,2, *}$ \\ ${ }^{1}$ Centro del Agua para América Latina y el Caribe, Tecnológico de Monterrey, Monterrey \\ 64849, Mexico \\ ${ }^{2}$ Departamento de Física, Tecnológico de Monterrey, Monterrey 64849, Mexico \\ * corresponding author: contreras.flavio@itesm.mx
}

\begin{abstract}
The stress-strain anisotropy -including both elastic properties and dislocation distributions - of crystalline systems can be studied using a dislocation model proposed by Ungár and Borbély. However this model requires fundamental parameters (i.e., contrast factors), which take into account both the elastic properties and symmetry for each system. In this study, such contrast factors were calculated from first principles for cubic slip-systems through the evaluation of the distortion tensor in a dislocation-dependent coordinate set. Moreover, a straightforward expression for the computation of contrast factors is provided. Further analysis of the microstructural parameters was carried out using the modified Williamson-Hall method to characterize rock-salt materials.
\end{abstract}

Key Words: X-ray line profile analysis · Strain anisotropy · Dislocation field · Contrast factor · Williamson-Hall

\section{Introduction}

Diffraction methods are commonly used to characterize the microstructure of materials. X-ray diffractometry, for example, is a powerful tool to study the shape, size and distribution of crystallites; lattice faults and twinings; and the arrangement and density of stress-strain dislocations $\left[\begin{array}{l}1-3] \\ 3\end{array}\right.$ All the above information is simultaneously embedded within the sample's diffractogram, and thus several approaches to estimate apparent size parameters and mean square strain values have been proposed along the last few decades. The Williamson-Hall (WH) and the Warren-Averbach (WA) are two classical methods [4 8 that can describe the microstructure for bulk materials. However, several assessments to obtain microstructural information resulted in the lack of a monotonic behavior as evidenced mainly for WH 911 . To effectively decouple the sample's size and strain contributions, Ungár and Borbély [12,13 modified the WH and WA methods making use of contrast factors $\left(C_{h k l}\right)$ which account for the stress-strain anisotropy introducing scaling factors. As a result, the accuracy and agreement of the microstructural parameters estimated by diffraction techniques is highly improved, and even can be compared to those measurements carried out by transmission electron microscopy [14].

A fundamental step to implement the proposed gauge relies on the evaluation of the distortion tensor in an anisotropic medium. By making use of the Lekhnitskii complex potential 15 16], the Stroh dislocation eigenvalues 17] and a dislocation-dependent coordinate system, a straightforward expression for the computation of this parameter can be obtained. Thus, the elastic component of dislocation contrast factors can be evaluated in the system's proper coordinates. In particular, the rock-salt $(\mathrm{Fm} \overline{3} m)$ structures im- 
pose additional restrictions on their dislocation allowance mainly due to the crystal's overall neutral charge. Even though a parametric evaluation of contrast factors on cubic symmetries has been previously implemented [18], its application does not transition directly into materials with more limiting degrees of freedom. Therefore the use of a first principles approach to calculate contrast factors for such materials is necessary, with different constraints arising for each symmetry/slip-system coupling.

Herein, the distortion tensor for cubic slipsystems is described using a stretched coordinate system that allowed to compute the elastic component of the contrast factors on each dislocation in a more simple way. In particular, the contrast factors for binary Fm $\overline{3} \mathrm{~m}$ systems are calculated from first principles and used to characterize the microstructure of $\mathrm{KCl}$ and $\mathrm{NaCl}$ materials through a modified WH analysis.

\section{Theoretical basis}

\section{Modified WH method}

The classic WH method [8] resolves both size $\left(\beta_{p}\right)$ and strain $\left(\beta_{s}\right)$ broadening contributions on real crystals by taking advantage of their different order dependence with respect to Bragg's angle $(\theta)$. The former contribution occurs due to the finite size effects of the diffracting system, while the later one arises from its lattice distortions. More specifically,

$$
\tilde{\beta}=\frac{1}{\tau}+2 \zeta \tilde{d}
$$

where $\tilde{\beta}=\beta \cos (\theta) / \lambda$ and $\tilde{d}=2 \sin (\theta) / \lambda$ are respectively the sample-related broadening and plane spacing, described in a reciprocal space and $\lambda$ is the source wavelength; $\tau$ is the apparent crystallite size as originally defined by Jones 4] while $\zeta$ is the apparent strain as originally defined by Stokes and Wilson [5]. Both $\tau$ and $\zeta$ are the integral breaths of the sample's crystallite size and the tensile strain distributions, respectively. In particular, $\tau$ was interpreted by Hall [6] as a characteristic length scale for the lattice regions which diffract coherently within the system, and $\zeta$ depends directly on the distribution curve which governs the system's strain. If the distribution is for example uniform and isotropic, $\zeta=2 \epsilon$, where $\epsilon$ is the maximum relative displacement $(\Delta d / d \equiv \Delta \tilde{d} / \tilde{d})$ of a lattice point from its ideal position. The modified WH method [12] broadens the scope of the original approach into systems on which strain anisotropy is significant by proposing a proper scaling factor $\delta=\tilde{d} \sqrt{C}$ instead of $\tilde{d}$ as in eq. (1), with $C$ being the average dislocation contrast factor. More significantly, $\tilde{\beta}(\delta)$ is to take a quadratic form as in

$$
\tilde{\beta}(\delta)=\tilde{\beta}_{0}+\tilde{\beta}_{1} \delta+\tilde{\beta}_{2} \delta^{2}
$$

where $\tilde{\beta}_{0} \equiv 1 / \tau^{\prime}, \tilde{\beta}_{1} \equiv 2 \zeta^{\prime}$ and $\tilde{\beta}_{2} \propto \sqrt{Q}$, where $\tau^{\prime}$ and $\zeta^{\prime}$ are the modified WH parameters and $Q$ is the the correlation coefficient between adjacent lattice points, often interpreted as the fluctuation $Q=\left\langle\rho^{2}\right\rangle-\langle\rho\rangle^{2}$ of the dislocation density, $\rho,[19$. In particular, eq. (2) reduces to a linear case as in eq. (1) when $Q$ is zero or negligible.

\section{Contrast Factors}

The computation of the average contrast factor $C$ from each individual contrast factor $C_{h k l}$, as extensively described by Armstrong and Lynch [20], can be performed as:

$$
\begin{aligned}
C \equiv\left\langle C_{h k l}\right\rangle & =\frac{1}{N} \sum_{i=1}^{N} C_{h k l}^{i} \\
& =\frac{1}{N} \sum_{K, L=1}^{6} \sum G_{K L}^{i} E_{K L}^{i}
\end{aligned}
$$

where $K, L$ are the indices for the reduced form of each 4-rank tensor and $N$ is the total number of degenerate slip systems (see Appendix 1). If not all slip-systems are equally populated, appropriate weight factors for each system should be calculated and included to the overall ensemble 20]. Specifically, the RHS of eq. (3) is split into a geometric component $\boldsymbol{G} \equiv G_{K L}=G_{i j k l}$ and an elastic one $\boldsymbol{E} \equiv E_{K L}=E_{i j k l}$, respectively described by:

$$
G_{i j k l}=\gamma_{i} \gamma_{j} \gamma_{k} \gamma_{l}
$$

and

$$
E_{i j k l}=\frac{1}{\pi} \int_{0}^{2 \pi} T_{i j} T_{k l} d \varphi
$$

with $\gamma_{i}$ being the direction cosine between the scattering vector and the slip coordinate system for a particular geometry and dislocation type (see [20]) and $T_{i j}$ is the distortion tensor associated with the displacement field of each dislocation.

The calculation of the geometric components for each contrast factor is relatively straightforward $[17$ and thus it is the evaluation of eq. (5) which turns out computationally demanding. Even though most calculations of contrast factors as pioneered by Borbely et.al. make use of numerical methods to perform this task [21], an analytical form for the distortion tensor can be obtained by introducing a 'stretched' coordinate system which readily integrates information about a particular dislocation - this within the Stroh-Lekhnitskii 
formalism. Moreover, $T_{i j}$ can be written as a linear combination of the distortion displacements, which take a closed, symmetric form when expressed in this 'stretched' coordinates as will be described next.

\section{Displacement Field}

In order to evaluate eq. (5), both the system's distortion tensor $T_{i j}$ within the dislocation plane and the overall $3 \mathrm{D}$ displacement field $u_{\kappa}$ must be evaluated. The later has been previously described by Teodosiu, with an explicit form given by 15$]$ :

$$
u_{\kappa}\left(x_{1}, x_{2}\right)=\frac{1}{\pi} \operatorname{Im}\left\{\sum_{\alpha=1}^{3} A_{\kappa, \alpha} D_{\alpha} F_{\alpha}\right\}
$$

with $F_{\alpha}=\ln \left(x_{1}+p_{\alpha} x_{2}\right)$ being the Lekhnitskii complex potential and $p_{\alpha}$ being the correspondent root of the sextic polynomial associated with each slip system, while $A_{\kappa, \alpha}$ and $D_{\alpha}$ are the eigenvalues to the corresponding Stroh eigenvalue equation associated with each dislocation. By realizing the complex nature of $A, D$, and $F$ (i.e. their real and complex components), an explicit form for the displacement field can be written as:

$$
\begin{gathered}
u_{\kappa}=\frac{1}{\pi} \sum_{\alpha=1}^{3}\left\{A_{\kappa, I} D_{R} F_{R}+A_{\kappa, R} D_{I} F_{R}\right. \\
\left.+A_{\kappa, R} D_{R} F_{I}-A_{\kappa, I} D_{I} F_{I}\right\}
\end{gathered}
$$

where the $X_{R}$ and $X_{I}$ subscripts denote real and imaginary part, respectively. The $\alpha$ subscript for each term of the equations above has been dropped for simplicity.

\section{Distortion Tensor in a stretched coordinate system}

An analytical form for the dislocation tensor can be obtained introducing a stretched coordinate system $\left(x_{1}, x_{2}\right),(r, \varphi) \leftrightarrow(\xi, \eta),(\rho, \phi)$ based upon each Stroh dislocation eigenvalue $p_{\alpha}=a+i c$ (see Fig. 1), with:

$$
\begin{cases}\xi=x_{1}+a x_{2} & \rho^{2}=\xi^{2}+\eta^{2} \\ \eta=c x_{2} & \phi=\arctan (\eta / \xi)\end{cases}
$$

and the corresponding transformation relation:

$$
\left(\begin{array}{l}
\xi \\
\eta
\end{array}\right)=\left(\begin{array}{ll}
1 & a \\
0 & c
\end{array}\right) \cdot\left(\begin{array}{l}
x_{1} \\
x_{2}
\end{array}\right)
$$

In this new coordinate system, the real and imaginary parts for the distortion displacement
A

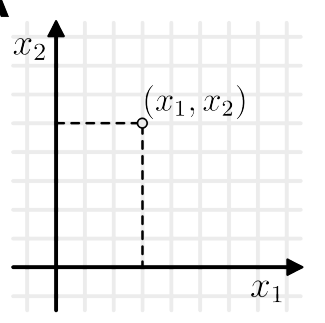

B

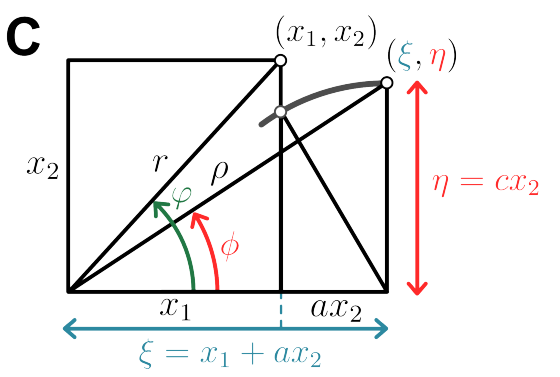

Figure 1: Cartesian (A) and 'Stretched' (B) coordinate axes system for a particular Stroh dislocation eigenvalue $p_{\alpha}=a+i c$. (C) Geometrical relationships between both coordinate systems.

$H_{i}=r \partial_{i} F$ with $\partial_{i} F=\frac{\partial F}{\partial x_{i}}$ and $\imath=r / \rho^{2}$ can be succinctly written as:

$$
\begin{aligned}
H_{1, R}=\xi \imath & H_{2, R}=(a \xi+c \eta) \imath \\
H_{1, I}=-\eta \imath & H_{2, I}=(c \xi-a \eta) \imath
\end{aligned}
$$

Again, the $\alpha$ subscripts were dropped for simplicity. Under the previous definitions, the close-form equation for the distortion tensor is given by:

$$
\begin{aligned}
T_{i j}= & \frac{2 \pi r}{b} \partial_{j} u_{i} \\
= & \frac{2}{b} \sum_{\alpha=1}^{3}\left\{A_{i, I} D_{R} H_{j, R}+A_{i, R} D_{I} H_{j, R}\right. \\
& \left.\quad+A_{i, R} D_{R} H_{j, I}-A_{i, I} D_{I} H_{j, I}\right\}
\end{aligned}
$$

It can be shown (see Appendix 2) that the distortion displacement (and thus the distortion tensor) can be expressed as a function of the polar angle $\varphi$ only. Thus, eq. (5) can equivalently defined in terms of the 'stretched' polar angle as:

$$
E_{i j k l}=\frac{1}{\pi} \int_{0}^{2 \pi} T_{i j} T_{k l} d \phi
$$

Finally, the eigenvalues $A_{\kappa, \alpha}$ and $D_{\alpha}$ are quantities which directly depend on the sample's elastic constants, and they can be directly calculated from the reduced elastic compliances and $p_{\alpha}$ 20. 


\section{Application to binary ( $\mathrm{Fm} \overline{3} \mathrm{~m})$ structures}

\section{Materials and methods}

Crystalline samples of $\mathrm{KCl}$ and $\mathrm{NaCl}$ were prepared by hand-milling in an agathe mortar. To remove any adsorbed water a subsequent heat treatment was performed at $450^{\circ} \mathrm{C}$ during $48 \mathrm{~h}$. For diffraction measurements the powders were deposited on a silicon plate. A ready-made plaque of crystalline $\mathrm{VO}$ was also measured for comparison.

X-ray diffraction measurements were performed on a Bruker X-ray D2 Phaser powder diffractometer using $\beta$-filtered, $\mathrm{Cu}-K_{\alpha}$ radiation $(\lambda=0.154$ $\mathrm{nm} ; 30 \mathrm{kV}, 10 \mathrm{~mA})$. Data were recorded with a step size of $0.010^{\circ}$ and an integration time of $1 \mathrm{~s}$ from $20^{\circ}$ to $100^{\circ}(2 \theta)$. The instrumental broadening was estimated with a corundum $\left(\mathrm{Al}_{2} \mathrm{O}_{3}\right)$ standard with measured instrumental function parameters of $u=8.2 \times 10^{-2}, v=-3 \times 10^{-4}$ and $w=8.0 \times 10^{-6}$. The instrumental contribution was subtracted from the raw data before further analysis. The integral breaths estimations were performed with the EVA Diffract.Suite ${ }^{\mathrm{TM}}$ and the computation of contrast factors was performed with code written in MATLAB.

\section{Results and Discussion}

The recorded X-ray measurements for both the VO plaque and the powder samples are shown in Fig. 2 The diffractograms showed a high degree of crystallinity and a match to their respective COD information card 22. The calculated lattice constants were $0.45 \mathrm{~nm}, 0.63 \mathrm{~nm}$ an 0.56 $\mathrm{nm}$ for $\mathrm{VO}, \mathrm{KCl}$ and $\mathrm{NaCl}$, respectively, in good agreement with the compound's reported lattice parameters.

The measured integral breaths for all three samples are presented in Fig. 3 in the form of classical WH plots. On the one hand, the VO plaque showed a relatively linear behavior, in good agreement with the behavior described by the classical WH analysis (as in eq. 1). The ionic powder samples, on the other hand, seem to show a lesser monotonic behavior as evidenced in a lower coefficient of determination, particurlarly for $\mathrm{KCl}$, which has been previously associated with strain anisotropy 13. Therefore, a modified WH analysis for both samples was implemented.

The crystal structure for alkali halides - more commonly referred as rock-salts - has been extensively studied and characterized. Even though their structure takes a cubic symmetry and either the well known FCC of BCC atomic packing, the bond's ionic nature impose a symmetry pertur-

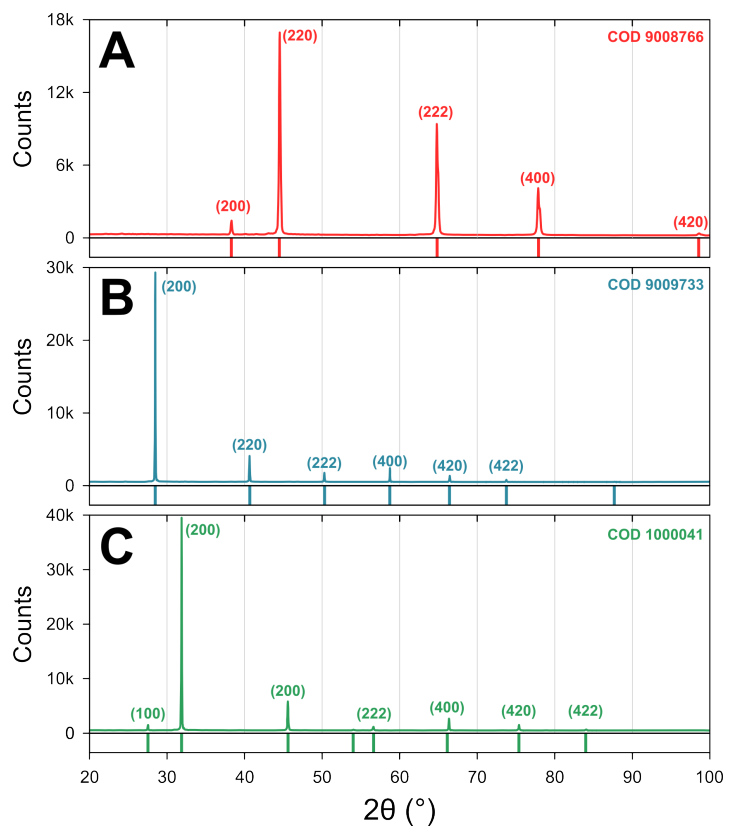

Figure 2: Recorded diffractograms for (A) a VO plaque, and powder samples of $(\mathrm{B}) \mathrm{KCl}$ and $(\mathrm{C}) \mathrm{NaCl}$. Their respective COD card is included at the bottom of each plot for reference.

bation and thus extra deformation restrains. A burgers vector $b=\frac{1}{2}\langle 100\rangle$, for example, is not symmetrically allowed as a half shift would compromise the crystal's overall neutrality, and thus the main burgers vector is given by $b=\frac{1}{2}\langle 110\rangle$. Such restriction then translates, for example, into the edge dislocations being forbidden for the primary slip system for alkali halides $\langle 110\rangle\{110\}$, as the orthogonal relations between the slip direction, the burgers vector, and the dislocation axis system can not be simultaneously realized.

Table 1: Average Contrast Factors $C \equiv\left\langle C_{h k l}\right\rangle$ for both $\mathrm{KCl}$ and $\mathrm{NaCl}^{a}$

\begin{tabular}{ccccc}
\hline & \multicolumn{2}{c}{$\mathrm{KCl}$} & \multicolumn{2}{c}{$\mathrm{NaCl}$} \\
$(h k l)$ & Edge & Screw & Edge & Screw \\
\hline 100 & 0.1857 & 0.1007 & 0.1870 & 0.1334 \\
200 & 0.1857 & 0.1007 & 0.1870 & 0.1334 \\
220 & 0.4388 & 0.1631 & 0.1782 & 0.1388 \\
222 & 0.5232 & 0.1839 & 0.1810 & 0.1334 \\
400 & 0.1857 & 0.1007 & 0.1782 & 0.1388 \\
420 & 0.3477 & 0.1406 & 0.1796 & 0.1362 \\
422 & 0.4388 & 0.1631 & 0.1803 & 0.1347 \\
\hline
\end{tabular}

${ }^{a}$ The $c_{11}, c_{12}$, and $c_{44}$ values (in GPa) for

both compounds were taken from 23 

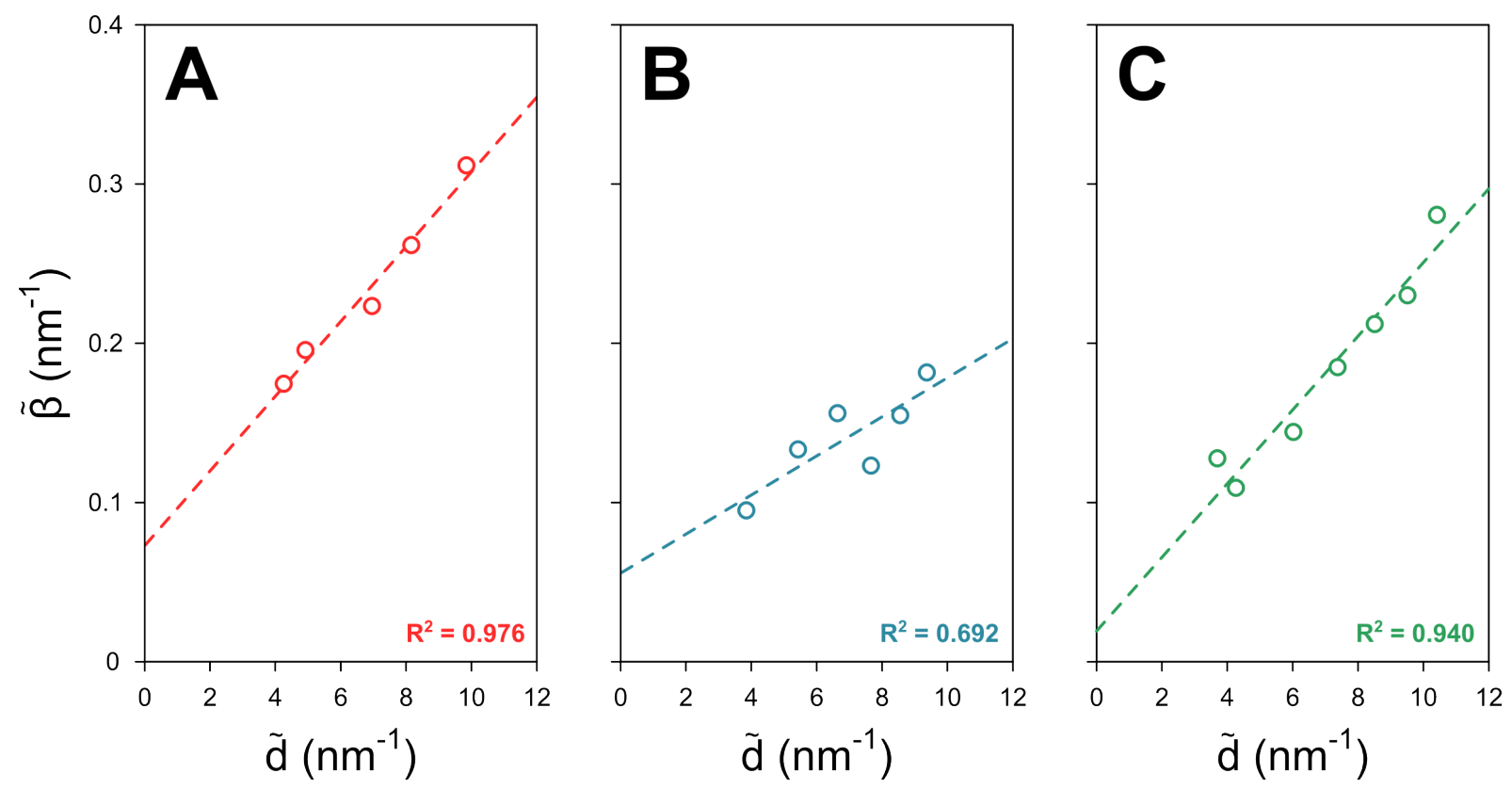

Figure 3: Classical WH plots in reciprocal space for (A) a VO plaque, and powder samples of (B) $\mathrm{KCl}$ and $(\mathrm{C}) \mathrm{NaCl}$.

In a similar manner, the screw dislocation is forbidden for the secondary $\langle 100\rangle\{110\}$ and tertiary $\langle 111\rangle\{110\}$ slip systems. While screw dislocations can have six different variants for the primary slip system, the orthogonal relationships between the burgers vector $(\hat{b})$, the dislocation line $(\hat{l})$ and the slip plane normal vector $(\hat{p})$ can not be simultaneously realized for edge dislocations. For example, in the case of the (110) plane and the [110] direction, $\hat{x_{3}} \| \hat{l}$ and $\hat{x_{2}} \| \hat{p}$. Because $\hat{b} \neq\langle 001\rangle$ in response to the crystal net charge neutrality, both $\hat{b}$ and $\hat{x_{1}} \|\langle 110\rangle$, and thus the $\hat{x_{1}} \perp \hat{x_{2}}$ can not be fulfilled for that slip plane-direction combination. An analogous argument applies to each permutation of $\langle 110\rangle\{110\}$, and thus edge dislocations are geometrically forbidden in this system. Overall, the primary slip system $\langle 110\rangle\{110\}$ allows only screw dislocations, while the secondary slip system $\langle 100\rangle\{110\}$ only permits edge dislocations to occur.

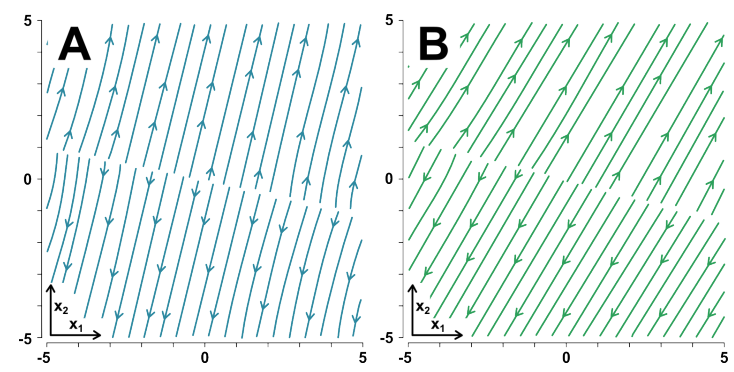

Figure 4: Graphical representations of the displacement field in the $\langle 100\rangle\{110\}$ edge slip system for (A) $\mathrm{KCl}$ and (B) $\mathrm{NaCl}$.
The contrast factors for the allowed edge and screw slip-systems for both $\mathrm{KCl}$ and $\mathrm{NaCl}$ were computed with eqs. (3) by using the elastic constants of both ionic compounds as reported in [23]. These results are shown in Table 1. The calculations for $\mathrm{KCl}$ suggest the (111) as the most favorable plane for dislocation, closely followed by (110). It is worth noticing that the obtained contrast factors for $\mathrm{NaCl}$ are significantly similar to each other, thus sugesting no overall preference for one plane against the other and that the dispersion in the integral breaths (i.e. divergence from theory) might be related to factors beyond strain anisotropy theory, such as those derived from plane faulting or twinning effects 13 . The displacement fields for both $\mathrm{KCl}$ and $\mathrm{NaCl}$ powder samples are presented in Fig. 4. In particular, the $\langle 100\rangle\{110\}$ edge slip system on a dislocation coordinate system in which the $x_{3}$-axis is parallel to the dislocation line is showed. The plots exemplify the way on which the sliding lattice blocks move in opposite directions, with the lower and upper halves moving in and out of the page, respectively. As observed the $\mathrm{KCl}$ field exhibits a slight curvature on the edges, in agreement with the contrast factors calculated which showed a slight anisotropy. The $\mathrm{NaCl}$ field lines are overall mostly parallel and all are practically identical, in accordance with the contrast factors being nearly identical for different $(h k l)$ reflections.

Then, the calculated contrast factors were used to introduce a proper scaling factor for the reciprocal plane spacing and perform a modified WH analysis for both salts. The best data fits were 

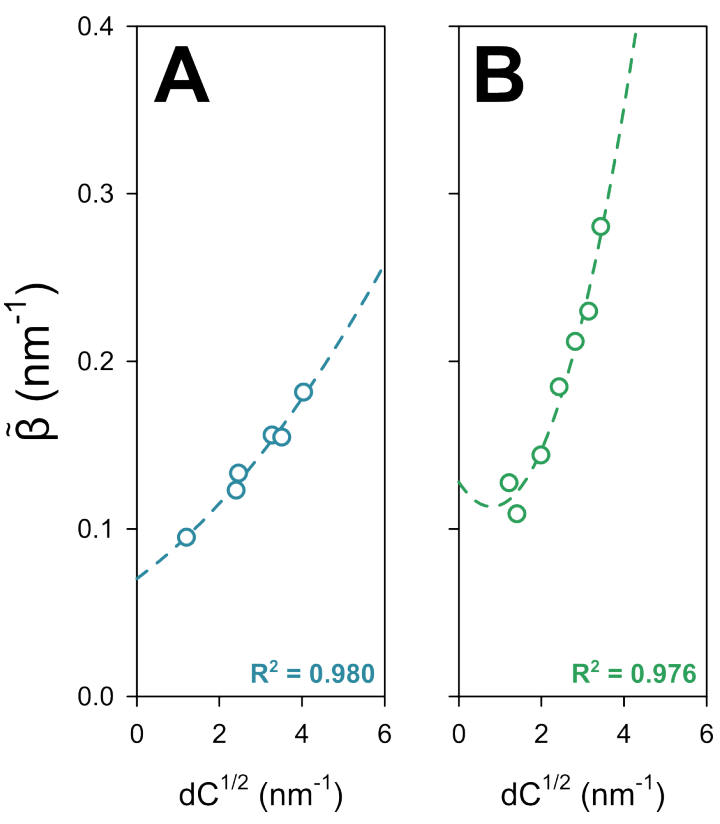

Figure 5: Modified WH plots in reciprocal space for powder samples of (A) $\mathrm{KCl}$ and (B) $\mathrm{NaCl}$.

obtained when the overall average contrast factors were calculated by assuming an equal proportion of screw/edge dislocations, thus suggesting that there is no preference for one dislocation type over the other. Both plots are presented in Fig. 5 and the corresponding fitting parameters are shown in Table 2 Both samples showed a decrease in the apparent crystallite size when comparing the modified WH parameters to their classical counterparts; surprisingly, the modified apparent strain for $\mathrm{NaCl}$ is compressive, while a regular $\mathrm{WH}$ analysis would describe a tensile strain.

On the one hand, the $\mathrm{KCl}$ fitting seems to be significantly improved from that of the classical analysis, with a relatively low $\tilde{\beta}_{2}$ coefficient (i.e. almost a linear behavior), corresponding to an anisotropic strain with a low fluctuation in the dislocation density. The $\mathrm{NaCl}$ fitting, on the other hand, reveals a more pronounced parabolic behavior with a $\tilde{\beta}_{2}$ coefficient being almost ten-fold as that of $\mathrm{KCl}$, thus suggesting a higher degree of

Table 2: Numerical fittings for both WH and Modified WH analysis of samples

\begin{tabular}{cccccc}
\hline & \multicolumn{2}{c}{ WH } & \multicolumn{3}{c}{ Modified WH } \\
& $\tau(\mathrm{nm})$ & $\zeta(\%)$ & $\tau^{\prime}(\mathrm{nm})$ & $\zeta^{\prime}(\%)$ & $\tilde{\beta}_{2}(\mathrm{~nm})$ \\
\hline $\mathrm{VO}$ & 13.68 & 1.18 & - & - & - \\
$\mathrm{KCl}$ & 17.86 & 0.74 & 14.22 & 0.90 & $2.2 \times 10^{-3}$ \\
$\mathrm{NaCl}$ & 52.08 & 1.37 & 7.80 & -1.85 & $2.2 \times 10^{-2}$ \\
\hline
\end{tabular}

correlation between the adjacent lattice points. The results obtained for $\mathrm{KCl}$ can be explained by the fact that the ionic bonds give rise to a lattice composed mainly by Ar cores on a large scale. This provides a more symmetric electronic environment, and thus a low degree of correlation is observed. Conversely, the $\mathrm{NaCl}$ lattice is composed of a mixture of $\mathrm{Ne}$ and $\mathrm{Ar}$ cores, and this pair coupling leads to a higher correlation degree.

\section{Conclusions}

The distortion tensor described in a stretched coordinate system allows for the straightforward computation of contrast factors for cubic slipsystems. Furthermore, the inclusion of symmetry constrains provides significant insights in the understanding of strain contributions. Within this context, this study extends the calculation of contrast factors to binary $\mathrm{Fm} \overline{3} m$ materials. For the first time, the contrast factors for $\mathrm{KCl}$ and $\mathrm{NaCl}$ were calculated and used to characterize their microstructure through the modified WH analysis.

\section{Acknowledgments}

Financial support from the National Council of Science and Technology of Mexico (CONACyT, grant 269399) is greatly appreciated.

\section{APPENDIXES}

\section{A1. Slip Systems}

A slip system is defined by a sliding plane $(h k l)$ which glides along or perpendicularly to a slip direction $[h k l]$. Each slip system can have a variety of realizations as defined by permutations of the plane and direction family components. For each material, both its geometry and atomic packing determine what particular combinations of planes and directions are most likely to undergo deformations. A primary slip system, for example, is that on which the coupled plane and direction carry simultaneously the largest planar and linear density, thus being the most likely to undergo deformations. A particular set of stress conditions, however, might lead to the expression of secondary or tertiary slip system for a particular deformation. Some geometries, however, even forbid some slip-system couplings to occur in the first place.

\section{A2. Distortion Displacement}

By expressing the relations in eq. 10 in either the polar coordinates $(r, \varphi)$ or the 'stretched' polar coordinates $(\rho, \phi)$, it can be easily shown that 
$\xi, \eta \propto r, \rho$ and $\imath \propto r^{-1}, \rho^{-1}$. Thus, the overall $r$ or $\rho$ dependence for the distortion displacement vanishes, and each component can be explicitly written as a function of $\varphi$ as given by:

$$
\begin{aligned}
H_{1, R}(\varphi) & =\frac{\cos (\varphi)+a \sin (\varphi)}{(\cos (\varphi)+a \sin (\varphi))^{2}+c^{2} \sin ^{2}(\varphi)} \\
H_{1, I}(\varphi) & =-\frac{c \sin (\varphi)}{(\cos (\varphi)+a \sin (\varphi))^{2}+c^{2} \sin ^{2}(\varphi)} \\
H_{2, R}(\varphi) & =\frac{a \cos (\varphi)+|p|^{2} \sin (\varphi)}{(\cos (\varphi)+a \sin (\varphi))^{2}+c^{2} \sin ^{2}(\varphi)} \\
H_{2, I}(\varphi) & =\frac{c \cos (\varphi)}{(\cos (\varphi)+a \sin (\varphi))^{2}+c^{2} \sin ^{2}(\varphi)}
\end{aligned}
$$

In particular, the relationship between $\varphi$ and $\phi$, as derived by using the definitions in eq. 8 is given by:

$$
\varphi=\arctan \left(\frac{\sin (\phi)}{c \cos (\phi)-a \sin (\phi)}\right)
$$

Thus, the distortion displacement can also be expressed as a function of the 'stretched' polar angle $\phi$ in a more symmetric way as given by:

$$
\begin{array}{rrr}
H_{1, R}(\phi) & =\cos (\phi) \cdot \Gamma(\phi) \\
H_{1, I}(\phi) & = & -\sin (\phi) \cdot \Gamma(\phi) \\
H_{2, R}(\phi) & = & (a \cos (\phi)+c \sin (\phi)) \cdot \Gamma(\phi) \\
H_{2, I}(\phi) & = & (c \cos (\phi)-a \sin (\phi)) \cdot \Gamma(\phi)
\end{array}
$$

$$
\text { with } \Gamma(\phi)=\sqrt{\left(\cos (\phi)-\frac{a}{c} \sin (\phi)\right)^{2}+\frac{1}{c^{2}} \sin ^{2}(\phi)}
$$

\section{References}

[1] J. Mittemeijer Eric and U. Welzel, Zeitschrift für Kristallographie 223, 552 (2008).

[2] P. Cordier, T. Ungar, L. Zsoldos, and G. Tichy, Nature 428, 837 (2004).

[3] V. Soleimanian and M. Mojtahedi, Applied Physics A 119, 977 (2015).

[4] F. W. Jones, Proceedings of the Royal Society of London A: Mathematical, Physical and Engineering Sciences 166, 16 (1938).

[5] A. R. S. Wilson and A. J. C, Proceedings of the Physical Society 56, 174 (1944).

[6] W. H. Hall, Proceedings of the Physical Society. Section A 62, 741 (1949).
[7] B. E. Warren and B. L. Averbach, Journal of Applied Physics 21, 595 (1950).

[8] G. K. Williamson and W. H. Hall, Acta Metallurgica 1, 22 (1953).

[9] V. Mote, Y. Purushotham, and B. Dole, Journal of Theoretical and Applied Physics 6, 1 (2012).

[10] S. G. Pandya, J. P. Corbett, W. M. Jadwisienczak, and M. E. Kordesch, Physica E: Low-dimensional Systems and Nanostructures 79, 98 (2016).

[11] Y. T. Prabhu, K. V. Rao, V. S. S. Kumar, and B. S. Kumari, World Journal of Nano Science and Engineering 4, 21 (2014).

[12] T. Ungár and A. Borbély, Applied Physics Letters 69, 3173 (1996).

[13] T. Ungár, Powder Diffraction 23, 125 (2008).

[14] T. Ungar, J. Gubicza, G. Ribarik, and A. Borbely, Journal of Applied Crystallography 34, 298 (2001).

[15] C. Teodosiu, Elastic models of crystal defects, Editura Academiei ; Springer-Verlag, Berlin ; New York, 1982.

[16] P. Klimanek and R. J. Kuzel, Journal of Applied Crystallography 21, 59 (1988).

[17] J. Martinez-Garcia, M. Leoni, and P. Scardi, Acta Crystallographica Section A 65, 109 (2009).

[18] T. Ungar, I. Dragomir, A. Revesz, and A. Borbely, Journal of Applied Crystallography 32, 992 (1999).

[19] I. Groma, T. Ungar, and M. Wilkens, Journal of Applied Crystallography 21, 47 (1988).

[20] N. Armstrong and P. Lynch, Determining the Dislocation Contrast Factor for X-ray Line Profile Analysis, pages 249-286, Springer, Berlin, Heidelberg, 2004.

[21] A. Borbely, J. Dragomir-Cernatescu, G. Ribarik, and T. Ungar, Journal of Applied Crystallography 36, 160 (2003).

[22] S. Grazulis et al., Journal of Applied Crystallography 42, 726 (2009).

[23] L. B. Bäckström and G, Physica Scripta 11, 43 (1975). 